\title{
Le concept de syndrome, en tant que construction didactique pertinente pour l'enseignement et l'apprentissage du savoir biomédical
}

\author{
The concept of syndrome, as a didactic construct relevant for biomedical \\ knowledge teaching and learning
}

La maîtrise du savoir biomédical, grâce aux connaissances et aux représentations que les médecins construisent à partir de lui, est l'une des ressources, nécessaire mais insuffisante, pour l'apprentissage du processus de raisonnement clinique (PRC) par l'étudiant en médecine ${ }^{[1-4]}$. En effet, le PRC est une démarche cognitive spécifique déployée par le clinicien face à un patient, qui n'implique pas explicitement le savoir biomédical, pour cette raison considéré comme «encapsulé ${ }^{[5]}$, en particulier chez les cliniciens experts ${ }^{[6]}$. C'est notamment le cas au cours du PRC centré sur l'élaboration du diagnostic, qui consiste à reconnaître et distinguer les maladies les unes des autres, au regard d'une catégorisation nosographique. Cette «encapsulation» du savoir biomédical, qui devient dès lors tacite, induit un certain nombre de difficultés chez l'étudiant en médecine dans ce contexte de l'apprentissage du PRC diagnostique $^{[7,8]}$, qui s'interroge notamment sur la place du savoir biomédical préalablement acquis et sur sa place dans le PRC, tant il est souvent difficile pour lui de l'articuler avec les données cliniques recueillies chez un patient.

Plusieurs propositions de modélisation des étapes du PRC ${ }^{[9-11]}$, ainsi qu'une taxonomie des difficultés que peuvent rencontrer les étudiants ${ }^{[12-14]}$, permettent d'entrevoir des réponses à cette question. Une meilleure connaissance des diverses représentations cognitives que construisent les experts (prototypes, axes et réseaux sémantiques, matrices probabilistes, etc.) et qu'ils mobilisent en situation de raisonnement clinique offre des pistes intéressantes pour envisager l'enseignement et l'apprentissage du raisonnement clinique. La notion de script (réseau organisé de connaissances et de représentations organisées, et utilisées pour la résolution d'un problème clinique) permet notamment de structurer le PRC diagnostique, à partir de descriptions orientées vers l'action de situations cliniques caractérisées ${ }^{[15,16]}$. Toutefois, ces approches pourraient être enrichies de concepts didactiques permettant d'établir des «ponts » entre le savoir biomédical et les données cliniques, en permettant de favoriser l'encapsulation de ce savoir par une structuration de celui-ci autour de notions théoriques ancrées au plus proche de la pratique clinique ${ }^{[1]}$. Dans le domaine du diagnostic, le concept de syndrome apparait tout à fait pertinent.

L'une des sources de difficulté majeure pour la manipulation par l'étudiant du savoir biomédical dans le contexte du diagnostic provient de la coexistence de deux grands types d'organisations distinctes pour les classifications des maladies : les classifica- 


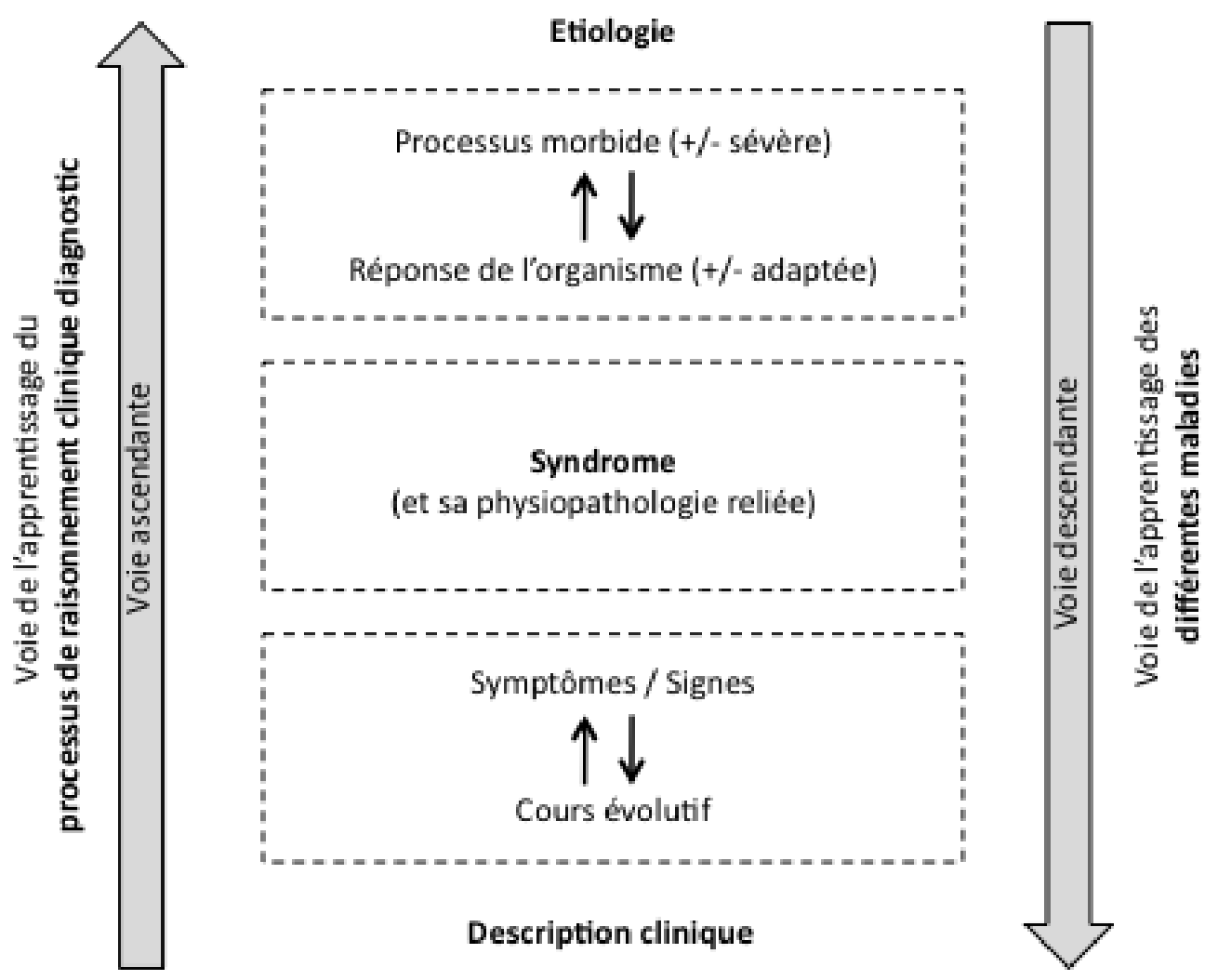

Fig. 1. Le syndrome comme proposition d'étage « intermédiaire » entre l'organisation phylogénétique (étiologie) et l'organisation phénotypique (description clinique) des maladies.

tions dites «phylogénétiques » et les classifications dites «phénotypiques » ${ }^{[17]}$. Les classifications «phylogénétiques » consistent à catégoriser les entités nosographiques selon des facteurs causaux. Ces classifications font référence à la définition princeps de la maladie comme « processus morbide » considérée comme une entité définissable par une cause caractérisée $^{[18]}$ et par une « réponse de l'organisme », plus ou moins adaptée. Les différentes classes étiologiques possibles sont : traumatique, toxique/médicamenteuse, métabolique, infectieuse, vasculaire, néoplasique, inflammatoire, dégénérative, génétique, ou idiopathique ${ }^{[9]}$. Cette organisation «phylogénétique » des maladies est très utile d'un point de vue « épistémique » et théorique, et s'inscrit ainsi efficacement dans la démarche de recherche scientifique clinique ou fondamentale ${ }^{[10]}$.
Les classifications «phénotypiques » consistent à regrouper les entités suivant des similitudes descriptives. Ces classifications font référence à une définition épidémiologique de la maladie qui est alors considérée comme un ensemble de caractéristiques sémiologiques, pronostiques et thérapeutiques communes. Bien qu'elles soient considérées comme transitoires et moins abouties que les classifications « phylogénétiques » (car non fondée sur une caractérisation étiologique ${ }^{[18]}$, ces classifications « phénotypiques » restent pertinentes pour structurer les pratiques cliniques $^{[10]}$.

Au cours des études médicales, les constructions didactiques en vue de l'apprentissage du socle de connaissances et des représentations biomédicales concernant les maladies se fonde généralement sur une classification «phylogénétique ». Chaque 
maladie est présentée suivant une « voie descendante » depuis sa cause spécifique jusqu'aux données cliniques la caractérisant. Or, cette « voie descendante » ne correspond pas au PRC diagnostique qui suivra un raisonnement inverse : une « voie ascendante » permettant l'identification de la maladie et de son étiologie à partir de la présentation de données cliniques et par l'intermédiaire notamment de la reconnaissance de script.

Les classifications phylogénétique et phénotypique sont progressivement encapsulées au sein de la démarche cognitive dans le cadre du PRC diagnostique expert. Pour l'étudiant en médecine, le concept de « syndrome » pourrait s'inscrire comme un élément intermédiaire entre classification "phylogénétique » et « phénotypique » afin de favoriser la compréhension par l'étudiant de ces deux logiques classificatoires (voir figure 1). Le « syndrome » se définit par un regroupement de signes et symptômes pertinents d'un point de vue phénotypique, permettant une économie et une efficacité cognitive pour le clinicien. Les syndromes sont en effet moins nombreux que l'ensemble des signes et symptômes possibles dans une situation clinique donnée mais également moins nombreux que l'ensemble des maladies. Pour autant, un syndrome présente généralement une physiopathologie bien identifiée (comme, par exemple, l'augmentation de la bilirubinémie dans le syndrome ictérique, ou le dysfonctionnement du système dopaminergique nigro-strié dans le syndrome parkinsonien) qui le situe au-delà d'un simple regroupement « phénotypique » purement clinique mais au dessous d'un regroupement «phylogénétique » fondé sur l'étiologie.

Il reste certainement à établir une classification médicale «phéno-phylogénétique » consensuelle des syndromes qui permettrait de structurer l'enseignement du savoir biomédical plus systématiquement autour de «l'étage syndromique », se situant entre le niveau des données cliniques et le niveau des maladies définies par leurs causes ${ }^{[19]}$. Cette présentation didactique pourrait permettre de favoriser l'intégration du savoir biomédical vers son encapsulation, accompagnant la conduite efficace du PRC diagnostique. Elle pourrait, en créant des liens entre « voie descendante » et « voie ascendante », diminuer les difficultés que les étudiants ont lorsqu'ils essayent d'utiliser méthodiquement leur savoir biomédical en commençant à s'entrainer au PRC diagnostique en situation clinique.

\section{Jean-Arthur MICOULAUD-FRANCHI $1,2,3$ *, Ali AMAD ${ }^{1,4}$, Pierre A. GEOFFROY ${ }^{1,5,6}$, Thomas FOVET ${ }^{1,4}$, Clélia QUILES ${ }^{1,7}$}

1 Association pour l'enseignement de la sémiologie psychiatrique (www.asso-aesp.fr)

2 Services d'explorations fonctionnelles du système nerveux, Clinique du sommeil, CHU de Bordeaux, Place Amélie Raba-Leon, 33076 Bordeaux, France

3 USR CNRS 3413 SANPSY, CHU Pellegrin, Université de Bordeaux, France ${ }^{4}$ Université de Lille, CHU Lille, Pôle de Psychiatrie, 59000 Lille, France

5 AP-HP, Groupe hospitalier Saint-Louis Lariboisière - F. Widal, Pôle de Psychiatrie et de Médecine Addictologique, 75475 Paris Cedex 10, France

${ }^{6}$ Université Paris Diderot, Sorbonne Paris Cité, UMR-S 1144, 75013 Paris, France

7 Université de Bordeaux, 33000 Bordeaux, France

\section{Références}

1. Woods NN. Science is fundamental: the role of biomedical knowledge in clinical reasoning. Med Educ 2007;41:1173-7.

2. Kulasegaram KM, Martimianakis MA, Mylopoulos M, Whitehead CR, Woods NN. Cognition before curriculum: rethinking the integration of basic science and clinical learning. Acad Med 2013;88:1578-85.

3. Kulasegaram K, Manzone JC, Ku C, Skye A, Wadey V, Woods NN. Cause and Effect: Testing a Mechanism and Method for the Cognitive Integration of Basic Science. Acad Med 2015;90(11 Suppl):S63-9.

4. Woods NN, Brooks LR, Norman GR. The value of basic science in clinical diagnosis: creating coherence among signs and symptoms. Med Educ 2005;39:107-12. 
5. Schmidt HG, Boshuizen HP. Encapsulation of biomedical knowledge. In: Evans D, Patel V, eds. Adavanced models of cognition for medical training and practice. New-York: Springer Verlag; 1992.

6. Schmidt HG, Rikers RM. How expertise develops in medicine: knowledge encapsulation and illness script formation. Med Educ 2007;41:1133-9.

7. Audetat MC, Laurin S, Sanche G. Aborder le raisonnement clinique du point de vue pédagogique. II. Les difficultés de raisonnement clinique à l'étape initial des données et de la génération d'hypothèses. Pédagogie Médicale 2011;12:231-6.

8. Sanche G, Audetat MC, Laurin S. Aborder le raisonnement clinique du point de vue pédagogique. III. Les difficultés de raisonnement clinique à l'étape du traitement et du raffinement des hypothèses : la fermeture prématurée Pédagogie Médicale 2012;13:103-8.

9. Charlin B, Lubarsky S, Millette B, et al. Clinical reasoning processes: unravelling complexity through graphical representation. Med Educ 2012;46:45463.

10. Pottier P, Planchon B. Les activités mentales au cours du raisonnement médical diagnostique. Rev Med Interne 2011;32:383-90.

11. Nendaz M, Charlin B, Leblanc V, Bordage G. Le raisonnement clinique : données issues de la recherche et implications pour l'enseignement. Pédagogie Médicale 2005;6:235-54.

12. Audetat MC, Laurin S, Sanche G. Aborder le raisonnement clinique du point de vue pédagogique. I. Un cadre conceptuel pour identifier les problèmes de raisonnement clinique. Pédagogie Médicale 2011; 12:223-9.

13. Audetat MC, Laurin S, Sanche G, et al. Clinical reasoning difficulties: a taxonomy for clinical teachers. Med Teach 2012;35:e984-9.

14. Bordage G. La prise de décision en médecine : quelques mécanismes mentaux et des conseils pratiques. Rev Med Interne 2005;26(S1):14-7.

15. Charlin B, Boshuizen HP, Custers EJ, Feltovich PJ. Scripts and clinical reasoning. Med Educ 2007;41: 1178-84.

16. Bordage G. Prototypes and semantic qualifiers: from past to present. Med Educ 2007;41:1117-21.

17. Micoulaud Franchi JA, Geoffroy PA, Amad A, Quiles C. Le jardinier et le botaniste. Proposition d'une organiation minimale de la sémiologie psychiatrique pour l'étudiant en médecine. Annales Medico Psychologiques 2015;173:460-69.

18. Paolaggi J, Coste J. Le raisonnement médical, de la science à la pratique clinique. Paris: ESTEM; 2001.

19. Micoulaud Franchi JA, Dumas G, Quiles C, Vion Dury J. [From clinic to the "foul and exciting field of life": A psychiatric point of view on clinical physiology]. Annales Medico Psychologiques 2017;175:70-85.

Correspondance et offprints : Jean-Arthur Micoulaud-Franchi. Mailto : jarthur.micoulaud@gmail.com 